\title{
The bond evolution mechanism of covalent sulfurized carbon during electrochemical sodium storage process
}

\author{
Tianjing $\mathrm{Wu}^{1}$, Chenyang Zhang ${ }^{1}$, Guoqiang Zou ${ }^{1}$, Jiugang $\mathrm{Hu}^{1}$, Limin $\mathrm{Zhu}^{2}$, Xiaoyu Cao ${ }^{2 *}$, \\ Hongshuai Hou ${ }^{1}$ and Xiaobo $\mathrm{Ji}^{{ }^{*}}$
}

\begin{abstract}
The excellent energy storage performance of covalent sulfur-carbon material has gradually attracted great interest. However, in the electrochemical sodium storage process, the bond evolution mechanism remains an elusive topic. Herein, we develop a one-step annealing strategy to achieve a high covalent sulfur-carbon bridged hybrid (HCSC) utilizing phenylphosphinic acid as the carbon-source/catalyst and sodium sulfate as the sulfur-precursor/salt template, in which the sulfur mainly exists in the forms of $\mathrm{C}-\mathrm{S}-\mathrm{C}$ and C-S-S-C. Notably, most of the bridge bonds are electrochemically cleaved when the cycling voltage is lower than $0.6 \mathrm{~V}$ versus $\mathrm{Na} / \mathrm{Na}^{+}$, leading to the appearance of two visible redox peaks in the following cyclic voltammogram $(\mathrm{CV})$ tests. The in-situ and $e x$-situ characterizations demonstrate that $\mathrm{S}^{2-}$ is formed in the reduction process and the carbon skeleton is concomitantly and irreversibly isomerized. Thus, the cleaved sulfur and isomerized carbon could jointly contribute to the sodium storage in 0.01-3.0 V. In a $\mathrm{Na}-\mathrm{S}$ battery system, the activated HCSC in cut off voltage window of 0.6-2.8 V achieves a high reversible capacity $\left(770 \mathrm{~mA} \mathrm{~h} \mathrm{~g}^{-1}\right.$ at $300 \mathrm{~mA} \mathrm{~g}^{-1}$ ). This insight reveals the charge storage mechanism of sulfur-carbon bridged hybrid and provides an improved enlightenment on the interfacial chemistry of electrode materials.
\end{abstract}

Keywords: one-step method, sulfur-carbon bridged complex, electrochemical mechanism, sodium storage

\section{INTRODUCTION}

Sulfur and carbon are abundant and environmentally benign, and the corresponding sulfur-containing carbon matrix derivatives, including sulfur-doped carbon $[1,2]$, sulfur/carbon composites [3,4], and sulfurized-carbon bridged materials $[5,6]$, etc., are widely utilized in new battery systems, such as room temperature $\mathrm{Na}$-ion batteries (SIBs) and Na-S batteries [7-9]. Among them, sulfur-doped carbon shows high reversible capacity in the SIB, which is mainly attributed to S doping with enlarged interlayer distance to facilitate the insertion and diffusion of $\mathrm{Na}^{+}[10,11]$. While the disordered carbon sulfur-doped with elemental $S$ as the sulfur source also shows high performance ( $333 \mathrm{~mA} \mathrm{~h} \mathrm{~g}^{-1}$ ) [1]. In fact, the energy storage mechanism of sulfur-doped carbon is an issue that is still debated $[1,7]$. The sulfur/carbon composites tend to be used in the Na-S battery system, especially in conjunction with sustainable energy sources $[9,12,13]$. And the whole reaction of $\mathrm{Na}-\mathrm{S}$ batteries can be represented as $\mathrm{S}_{8}+16 \mathrm{Na} \rightarrow 8 \mathrm{Na}_{2} \mathrm{~S}$ (theoretical specific energy, $1,672 \mathrm{~mA} \mathrm{~h} \mathrm{~g}^{-1}$ ) [12]. It has the potential to provide much higher energy density than traditional intercalation SIB electrodes, while the significant capacity decay is endemic, being in part caused by the shuttle mechanisms of the intermediate discharge products $[8,14,15]$. The sulfurized-carbon bridged complexes have moderate sulfur content, and the sulfur mainly exists in the forms of $\mathrm{C}-\mathrm{S}-\mathrm{C}$ and C-S-S-C. Inheriting the two properties, these materials could be applied in both Na-ion and Na-S systems [13]. Chen's group prepared sulfurized-carbon (SG) and investigated it as an anode for SIBs [16], showing a high reversible capacity of $291 \mathrm{~mA} \mathrm{~h} \mathrm{~g}^{-1}$. Lu's group used sulfurized-carbon (CSCM-29) in the Na-S system [5], obtaining a capacity of $678 \mathrm{~mA} \mathrm{~h} \mathrm{~g}^{-1}$. These materials have gradually attracted great interest in electrochemical energy storage systems. However, they are usually prepared utilizing $\mathrm{H}_{2} \mathrm{~S}$ gas, elemental $\mathrm{S}$, and sulfur-containing polymers as the sulfurizing reagents $[5,17]$.

\footnotetext{
${ }^{1}$ Key Laboratory of Powder Metallurgy, College of Chemistry and Chemical Engineering, Central South University, Changsha 410083, China

${ }^{2}$ College of Chemistry, Henan University of Technology, Zhengzhou 450001, China

* Corresponding authors (emails: xji@csu.edu.cn (Ji X); caoxy@haut.edu.cn (Cao X))
} 
These strategies involve complicated synthetic processes, and tend to result in low sulfur content and extra cost. Moreover, the deep and intricate universals for the electrochemical properties are still undistinguishable, because the charge/discharge products are difficult to detect and capture [8]. And the in-situ characterization platforms to track the reacted intermediates have not been widely wellestablished as yet.

Herein, utilizing phenylphosphinic acid and sodium sulfate as the starting materials, we prepared a highly sulfurized carbon bridged material (HCSC, S: 34.8 wt.\%) through the self-thermal carbonized reaction and industrial method for $\mathrm{Na}_{2} \mathrm{~S}$ production. The HCSC electrode shows unique electrochemical properties over varied voltage ranges of both discharge/charge. The Nastorage machanism of HCSC is as follows: in the reduction process, most of the $\mathrm{C}-\mathrm{S}$ and $\mathrm{S}-\mathrm{S}$ bonds are electrochemically cleaved when the discharging voltage is below $0.6 \mathrm{~V}$, and the intermediate $\mathrm{Na}_{2} \mathrm{~S}$ is simultaneously formed. Upon oxidation, high charging potential (close to $3.0 \mathrm{~V}$ ) leads to the transformation of cleaved-S into sodium polysulfide and elemental sulfur; meanwhile it results in the isomerization of carbon skeleton. Finally, the following charging/discharging process principally manifests as the electrochemical performances of both elemental sulfur and the isomerized carbon. Therefore, after being activated at $0.01-3.0 \mathrm{~V}$, the HCSC electrode applied in the Na-S battery system $(0.6-2.8 \mathrm{~V})$ delivers a high reversible capacity $\left(770 \mathrm{~mA} \mathrm{~h} \mathrm{~g}^{-1}\right.$ over 200 cycles at $\left.300 \mathrm{~mA} \mathrm{~g}^{-1}\right)$ and a superior rate capability $\left(590 \mathrm{~mA} \mathrm{~h} \mathrm{~g}^{-1}\right.$ at $3,200 \mathrm{~mA} \mathrm{~g}^{-1}$ ).

\section{EXPERIMENTAL SECTION}

\section{Preparation of materials}

Phenylphosphinic acid $(1.5 \mathrm{~g})$ was homogeneously mixed with sodium sulfate $(12.0 \mathrm{~g})$ by planetary ball-milling. The mixture was annealed at $600^{\circ} \mathrm{C}$ for $2 \mathrm{~h}$ under an Ar atmosphere and then the resultant brown solid (HCSC) was easily collected through dissolution and centrifugation, which was then rinsed with anhydrous ethanol and deionized water, followed by drying at $70^{\circ} \mathrm{C}$ for $12 \mathrm{~h}$. In the same way, another similar organic compound (tetraethyl[1,4-phenylenebis(methylene)]bisphosphonate) could be also used as the starting material to produce analogous sulfurized-cabon (BP-SC). In comparison with the compounds mentioned above, the sulphur doped carbon (BC$\mathrm{SC})$ was prepared with benzoic acid as the starting material. Given the above, the organic functional group of $\mathrm{C}-\mathrm{PO}_{3} \mathrm{R}_{2}$ is beneficial to the sulfonation of carbon.

\section{Condition experiments}

In order to obtain the material with the best comprehensive properties, the ratio of raw materials and the reaction temperature were optimized via a series of experiments. The sulfurized-carbon of HCSC-F and HCSC-T were prepared according to the similar process for preparing HCSC, except that the weight ratio of phenylphosphinic acid/ $\mathrm{Na}_{2} \mathrm{SO}_{4}$ (1:4 and 1:12) was adjusted. HCSC-50 and HCSC-70 were prepared at 500 and $700^{\circ} \mathrm{C}$, respectively. The corresponding X-ray photoelectron spectroscopy (XPS) results are displayed in Table S1 and Fig. S1.

\section{The capture of intermediates (D-BC, C-IC, and C-YS)}

$E x$-situ experimental techniques were employed to capture the intermediates in the first cycle. The fully discharged electrode slices were extracted with alcohol and distilled water, and the mother liquor was mixed with a $\mathrm{CuSO}_{4}$ solution to produce black sediment that was washed with water and alcohol for three times to obtain black solid (D-BC). The final charged HCSC electrode was extracted in chloroform; both the black precipitate and mother liquor were reprocessed. On one hand, after the extracted residue was washed with water and chloroform for three times, the purified black C-IC was obtained through drying at $70^{\circ} \mathrm{C}$ under a vacuum for $12 \mathrm{~h}$. On the other hand, the mother solution was dried by rotary evaporation; the residuum was cleaned with distilled water and alcohol to produce yellow solid C-YS.

\section{RESULTS AND DISCUSSION}

\section{Synthesis and characterization}

The additional bonding of $\mathrm{C}-\mathrm{P}$ is beneficial to constructing the $\mathrm{S}-\mathrm{C}-\mathrm{P}$ moiety and forming the analogues of Lawesson's reagent $[18,19]$, which could promote the reactivity for sulfonation. Coupled with the industrial method for $\mathrm{Na}_{2} \mathrm{~S}$ production $\left(\mathrm{Na}_{2} \mathrm{SO}_{4}+2 \mathrm{C} \rightarrow \mathrm{Na}_{2} \mathrm{~S}+\right.$ $\mathrm{CO}_{2}$ ), we report an effective method to prepare the HCSC as illustrated in Fig. 1a. Briefly, phenylphosphinic acid $\left(\mathrm{C}_{6} \mathrm{H}_{5}-\mathrm{PO}_{3} \mathrm{H}_{2}\right)$ is gradually carbonized to form an active carbon matrix, which chemically reacts with $\mathrm{Na}_{2} \mathrm{SO}_{4}$ to produce $\mathrm{Na}_{2} \mathrm{~S}$, in which $\mathrm{S}^{2-}$ can be captured by the available active sites on the carbon matrix, along with the synergistic effect of S-C-P $[20,21]$, leading to the formation of covalent sulfurized-carbon. With the optimized conditions (Table S1 and Fig. S1), the HCSC has the most covalent sulfur at $>22$ at. $\%$ on the surface. In the same condition, the similar organic compound (tetraethyl[1,4phenylenebis(methylene)]bis-phosphonate) could be also 


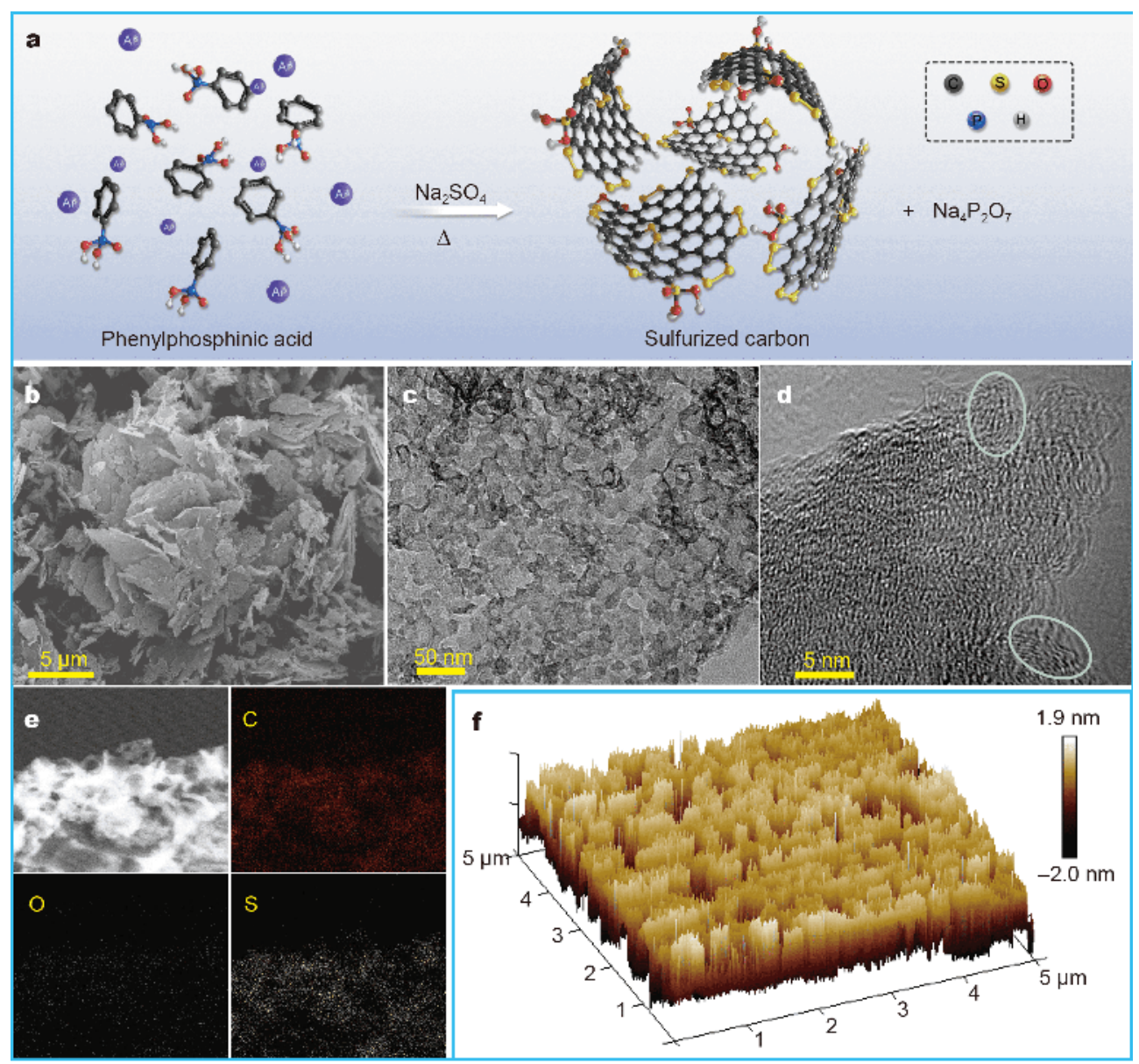

Figure 1 Preparation and characterization of HCSC. (a) Schematic illustration of the experimental procedure. (b-d) SEM, TEM and HRTEM images of partially graphitized HCSC. (e) Elemental mapping results. (f) AFM stereogram.

used to produce analogous sulfurized-cabon (BP-SC), and the covalent sulfur content is about 17 at.\% (Fig. S2). In comparison with HCSC and BP-SC, the sulfur doped carbon BC-SC (S: 1.75 at.\%) was prepared with benzoic acid $\left(\mathrm{C}_{6} \mathrm{H}_{5}-\mathrm{COOH}\right)$ as the starting materials (Fig. S3e, f). According to the analysis of crude products, $\mathrm{S}^{2-}$ is detected from the coarse product of BS-SC (Fig. S3a, c), while $\mathrm{P}_{2} \mathrm{O}_{7}{ }^{4-}$ tends to be formed in the reaction mixture of HCSC (Fig. S3b, d). This result confirms that the $\mathrm{Na}_{2} \mathrm{~S}$ is generated in the carbonization process and the $\mathrm{C}-\mathrm{PO}_{3} \mathrm{R}_{2}$ is beneficial to the sulfonation of carbon.

From the transmission electron microscopy (TEM) and high-resolution TEM (HRTEM) images (Fig. 1b-d), an amorphous nature and partial graphitization of HCSC are observed. Powder X-ray diffraction (XRD) data was employed to refine the structure of disordered carbon (Fig. S4c). The elemental mapping results (Fig. 1e) unambiguously demonstrate that $\mathrm{C}, \mathrm{S}$, and a small amount of elemental $\mathrm{O}$ are uniformly distributed in the framework of HCSC. Moreover, the elemental analysis further proves that the accurate weight contents of $\mathrm{C}, \mathrm{O}, \mathrm{S}$, and $\mathrm{H}$ within the HCSC composite are 56.7\%, 6.6\%, 34.8\%, and $1.5 \%$, respectively, and the contents of other heteroatoms ( $\mathrm{N}$ and $\mathrm{P}$ ) are less than $0.5 \%$ (Fig. S4j), agreeing well with the aforementioned XPS analysis. The Raman spectroscopy reveals four remarkable peaks (Fig. S4d): two peaks are located at 361 and $496 \mathrm{~cm}^{-1}$, corresponding to the stretching vibration of $\mathrm{C}-\mathrm{S}-\mathrm{C}$ and $\mathrm{C}-\mathrm{S}-\mathrm{S}-\mathrm{C}$ bonds $[22,23]$, respectively. At 1,360 and $1,550 \mathrm{~cm}^{-1}$, the low $I_{D} /$ $I_{\mathrm{G}}$ ratio (0.47) corroborates with the HRTEM, further implying the partial graphitization of HCSC structure [24-26]. Atomic force microscopy (AFM) indicates HCSC with a thickness of $\sim 2 \mathrm{~nm}$ (Fig. 1f and Fig. S4a, b). There exist a variety of sized holes across its surface, which is responsible for its corresponding high surface area $\left(396.6 \mathrm{~m}^{2} \mathrm{~g}^{-1}\right)$ (Fig. S4i). In the full survey XPS 
spectrum (Fig. S4g), the predominant peaks of C 1s, O 1s, $S 2 p$, and $S 2 s$ are found at around $285,532,165$, and $229 \mathrm{eV}$, respectively [27,28]. The high resolution S 2p spectrum is presented in Fig. S4h, where three peaks are observed at binding energies of 163.7, 164.8 and $167.8 \mathrm{eV}$, which are attributed to the S-S, S-C and S-O covalent bonds, respectively [29]. Fourier transform infrared main chemical state of covalent-sulfur. In Fig. S4e, the thermogravimetric analysis (TGA) of HCSC was conducted under $\mathrm{Ar}$ and air atmosphere at a heating rate of $5^{\circ} \mathrm{C} \min ^{-1}$, respectively. The weight loss occurring between room temperature and $100^{\circ} \mathrm{C}$ corresponds to the loss of superficial water molecules. In the following region, the TGA curve becomes flat between 100 and $375^{\circ} \mathrm{C}$, indicating no elemental sulfur within the HCSC. Especially, in the Ar condition, the maximum weight loss occurs at $\sim 520^{\circ} \mathrm{C}$ (Fig. S4e-1). Upon raising temperature, the covalent sulfur, oxygen, and incomplete carbonized matrix will continue to deplete. All these results confirm that the chemical refined-S is contained in the HCSC sample mainly in the forms of $\mathrm{C}-\mathrm{S}-\mathrm{C}$ and $\mathrm{C}-\mathrm{S}-\mathrm{S}-\mathrm{C}$.

\section{Electrochemical sodium storage}

The fabricated batteries based on HCSC electrode display excellent cycling stability and rate capacity. As shown in Fig. 2a, the first cycle columbic efficiency of HCSC (based spectroscopy (FT-IR) image (Fig. S4f) further proves the

on the mass of HCSC, $\sim 2.0 \mathrm{mg}$ ) at $100 \mathrm{~mA} \mathrm{~g}^{-1}$ is $52 \%$ and quickly approaches $\sim 100 \%$ in subsequent cycles. The HCSC exhibits a stable cycling for over 150 cycles with specific capacity of $570 \mathrm{~mA} \mathrm{~h} \mathrm{~g}^{-1}$. For a long-term cycling test at high current density $\left(1,000 \mathrm{~mA} \mathrm{~g}^{-1}\right)$, a reversible specific capacity of $393 \mathrm{~mA} \mathrm{~h} \mathrm{~g}^{-1}$ is obtained with capacity decay of $\sim 0.03 \%$ per cycle up to 600 cycles (Fig. 2c). In addition to the high reversible capacity and long stability, Fig. 2b shows that the HCSC electrode retains its high reversible capacity across various rates. The electrode reaches stable capacity of 580, 550 and $473 \mathrm{~mA} \mathrm{~h} \mathrm{~g}^{-1}$ at 100,200 and $800 \mathrm{~mA} \mathrm{~g}^{-1}$, respectively (Fig. S5a). The high-rate capability indicates that the electrode material allows fast transfer of $\mathrm{Na}^{+}$and electrons. When the current rate is reduced back to $100 \mathrm{~mA} \mathrm{~g}^{-1}$ after the rate test, the initial capacity is restored. Moreover, a reversible capacity of $330 \mathrm{~mA} \mathrm{~h} \mathrm{~g}^{-1}$ could be obtained at a high current density of $3,200 \mathrm{~mA} \mathrm{~g}^{-1}$, which is higher than most of the reported sulfur doped carbon materials $[9,28,30,31]$. This means that the sodium storage performance can be enhanced by introducing covalent sulfur into carbon matrix. We also investigated the cycling life as a function of different active mass loadings (Fig. S5b), where lighter HCSC electrode exhibits higher reversible capacity and better cycling performance, agreeing well with the previous report [4].
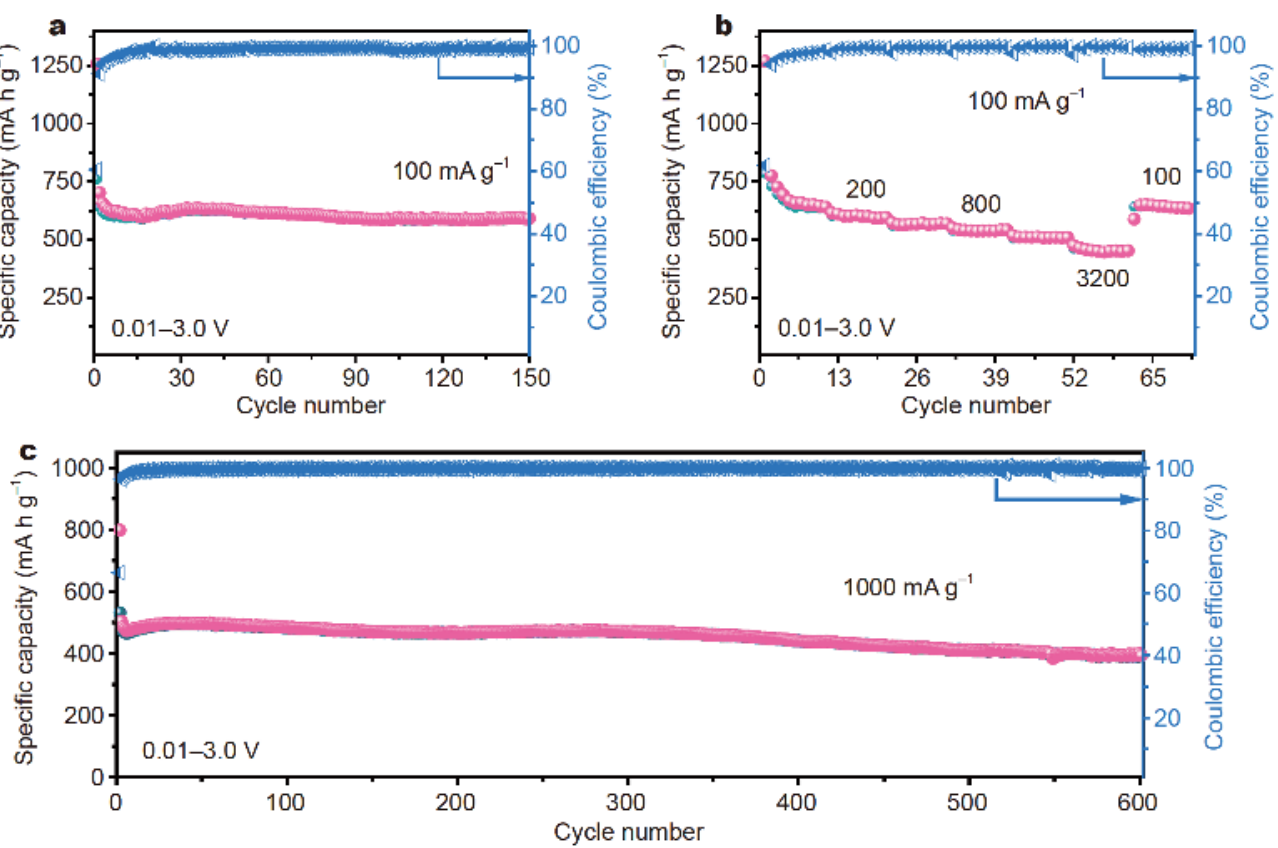

Figure 2 Electrochemical sodium storage in the potential window of $0.01-3.0 \mathrm{~V}$. (a) Cycling performance of the HCSC electrode at $100 \mathrm{~mA}^{-1}$. (b) Rate behaviors at different rates from 100 to $3,200 \mathrm{~mA} \mathrm{~g}^{-1}$. (c) Long-term cycling performance at $1,000 \mathrm{~mA} \mathrm{~g}^{-1}$. 
The evolution of cyclic voltammograms (CVs) and electrochemical impedance spectra (EIS)

Fig. 3 presents the CVs of HCSC electrode collected with different potential windows at the same scan rate of $0.2 \mathrm{mV} \mathrm{s}^{-1}$. The peaks in the CVs represent the redox reaction between HCSC and sodium. In Fig. 3a, the CV test was directly swept at the cut-off potential range of the $\mathrm{Na}-\mathrm{S}$ battery system $(0.6-3.0 \mathrm{~V})$, and there is no characteristic peak for sodium and sulfur reaction. Only a small reduction peak is observed at $\sim 1.2 \mathrm{~V}$, which is associated with the reaction between the $\mathrm{Na}^{+}$and surface functional groups [32]. Interestingly, as the potential scans down to $0.3 \mathrm{~V}$, a gentle redox peak appears at 1.4/1.8 V (Fig. 3b), which can be assigned to the redox reaction between $\mathrm{Na}$ and the chemically refined $\mathrm{S}$. Especially, in Fig. 3c, where the potential is further reduced to $0.01 \mathrm{~V}$, two pairs of reversible redox peaks are obviously located at $1.8 / 2.15 \mathrm{~V}$ and $1.1 / 1.8 \mathrm{~V}$. The redox couples remain stable when the potential window is shortened to $0.6-2.8 \mathrm{~V}$ (Fig. $3 \mathrm{~d}$ ), which is similar to the CVs of elemental sulfur $\left(\mathrm{S} \rightarrow \mathrm{S}_{x}{ }^{2-} \rightarrow \mathrm{S}^{2-}\right)$ [3]. The detailed CVs of HCSC electrode display the obvious influence of the potential range on the electrochemical behaviors (Fig. 3e and Fig. S6). Notably, when lithium is employed as the reference electrode, the analogous phenomenon is also observed (Fig. S8): when the CV of the HCSC electrode is scanned at the voltage region of Li-S batteries $(1.0-3.0 \mathrm{~V})$, no redox peak is found. The reversible oxi-
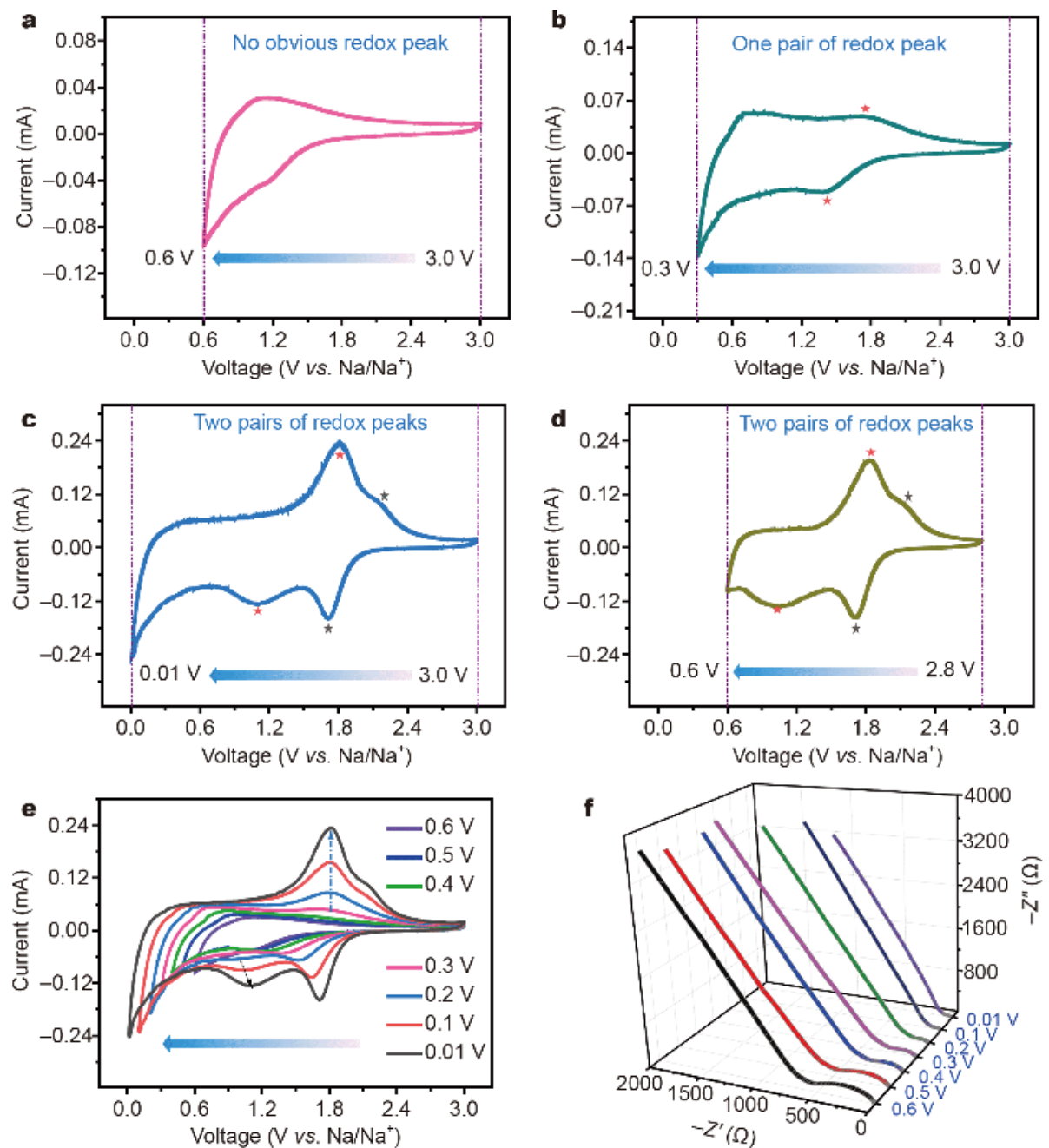

Figure 3 The CVs and Nyquist plots of HCSC electrode determined at different potential ranges: $0.6-3.0 \mathrm{~V}$ (a), 0.3-3.0 V (b) and 0.01-3.0 V (c), respectively. (d) After the final scan (c), the voltage range is shortened to the voltage window of the Na-S battery (0.6-2.8 V), in which the redox peaks of sulfur are still presented. (e) The low potential is decreased from 0.6 to $0.01 \mathrm{~V}$ at $0.2 \mathrm{mV} \mathrm{s}^{-1}$, and the redox peaks are gradually appeared. (f) EIS patterns. 
dation/reduction peaks gradually become apparent when the potential is decreased from 0.8 to $0.01 \mathrm{~V}$. Compared with the sodium electrode, the standard electrode potential of lithium is much lower due to the smaller radius, which allows the $\mathrm{Li}^{+}$to be easily intercalated into the HCSC, and thus the redox peak could be found when the low potential is reduced to $0.6 \mathrm{~V}$, rather than $0.3 \mathrm{~V}$ for sodium.

The EIS was collected to analyze the electrochemical behavior of the HCSC electrode during the cycles (Fig. 3f). In Fig. S7a, after scanning at 0.6-3.0 V, the fitting result is in good agreement with the measured data, demonstrating that the impedance spectrum is mainly composed of a semicircle at medium/high frequency ranges and a short slope tail at low frequency. In all curves, the high-frequency semicircle denotes the chargetransfer resistance and the sloping line in the low-frequency region represents the Warburg impedance. The charge-transfer resistance of HCSC decreases as the voltage range widens. And the Warburg impedance exhibits small variations, confirming the faster charge-transfer kinetics within the HCSC (Fig. S7b). These results posi- tively demonstrate that the host structure of HCSC is gradually altered as the potential stepwisely scans down to $0.01 \mathrm{~V}$, reflecting the electrochemical activation of covalent $\mathrm{C}-\mathrm{S}$ units.

\section{Cyclic performance evolution}

The galvanostatic discharge/charge $\left(100 \mathrm{~mA} \mathrm{~g}^{-1}\right)$ tests were further performed on the assembled SIB (based on the mass of HCSC). As shown in Fig. 4, the intercalation does not significantly alter the host structure, thus guaranteeing high reversibility upon cycling in $0.6-2.8 \mathrm{~V}$ with the capacity of $127 \mathrm{~mA} \mathrm{~h} \mathrm{~g}^{-1}$ (Fig. 4b). Subsequently, the low potential is scanned further to $0.01 \mathrm{~V}$, which results in two visible discharge $(\sim 1.75 \mathrm{~V}, \sim 0.8 \mathrm{~V})$ and charge plateaus $(\sim 1.8 \mathrm{~V})$, along with an increased discharge capacity (542 $\mathrm{mA} \mathrm{h} \mathrm{g}^{-1}$, Fig. 4c). Similarly, when the low voltage is reverted to $0.6 \mathrm{~V}$, the platforms still exist in the discharge/charge profile. And the discharge capacity ( $342 \mathrm{~mA} \mathrm{~h} \mathrm{~g}^{-1}$, Fig. $4 \mathrm{~d}$ ) is higher than the initial result (127 $\mathrm{mA} \mathrm{h} \mathrm{g}^{-1}$ ), which could be attributed to the excellent energy capacity of the Na-S battery (theoretical value: $760 \mathrm{~W} \mathrm{~h} \mathrm{~kg}^{-1}$ ) [7]. Thus, the corresponding battery per-
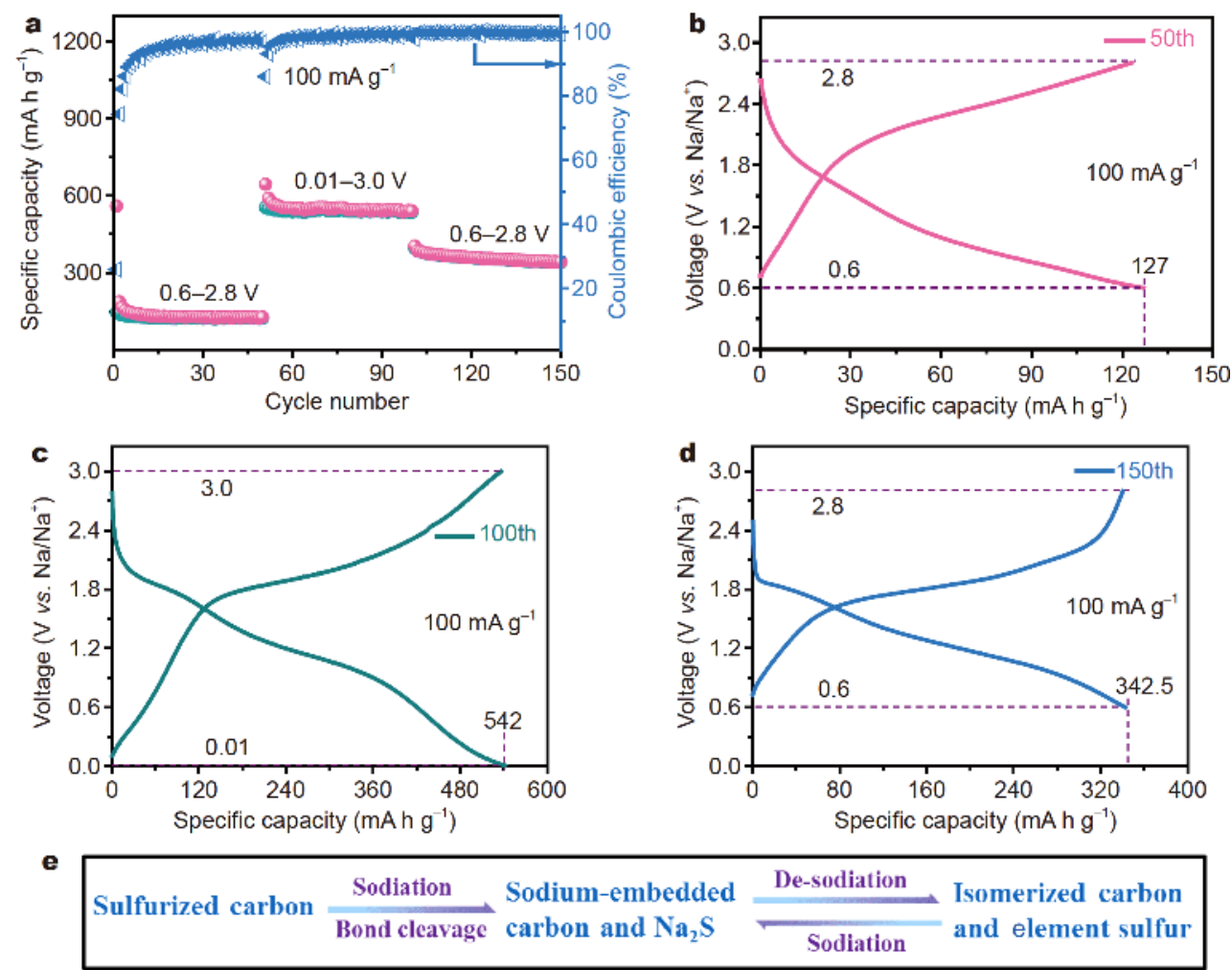

Figure 4 Cyclic performance evolution of HCSC at different potential windows. (a) Cycling performance of HCSC at $100 \mathrm{~mA} \mathrm{~g}^{-1}$ (based on the total mass of HCSC). (b-d) Discharge/charge curves. There is no obvious discharge plateau in the region of $0.6-2.8 \mathrm{~V}$ (b). After discharge to $0.01 \mathrm{~V}$, two discharge platforms could be observed (c) and they still exist as the potential window is reduced back to $0.6-2.8 \mathrm{~V}$ (d). (e) Possible reaction process. 
formance is well consistent with the $\mathrm{CV}$ results: as the discharge voltage is scanned down to $0.01 \mathrm{~V}$, the chemically refined $S$ is broken away from the carbon skeleton and then progressively oxidized to elemental $S$ in the charge process. Ultimately, the isomerized carbon and cleaved covalent-S would come together and contribute to the overall specific capacity. Fig. 4e shows the possible reaction process.

\section{Na-S battery behaviors}

Inspired by the above results, the performance of HCSC electrode in Na-S battery was further studied after being activated by $\mathrm{CV}$ scan at $0.01-3.0 \mathrm{~V}$. Apparently, the activated HCSC electrode exhibits stable cycling performance and excellent rate capabilities at $0.6-2.8 \mathrm{~V}$ (based on the mass of sulfur). As shown in Fig. 5a, The cathode delivers an initial discharge capacity of $1,157 \mathrm{~mA} \mathrm{~h} \mathrm{~g}^{-1}$ in the first cycle, with $983 \mathrm{~mA} \mathrm{~h} \mathrm{~g}^{-1}$ being reversible, which indicates excellent utilization of covalent-S. It still maintains a reversible capacity of $770 \mathrm{~mA} \mathrm{~h} \mathrm{~g}^{-1}$ with capacity decay of $0.16 \%$ per cycle over 200 cycles at $300 \mathrm{~mA} \mathrm{~g}^{-1}$. This electrochemical performance is superior to or comparable with previously reported results of sulfur- doped carbon materials as per Fig. 5d. Fig. 5b shows the rate capability of the HCSC electrode. It could deliver reversible capacities of $1,140,880$, and $590 \mathrm{~mA} \mathrm{~h} \mathrm{~g}^{-1}$ at 200,800 , and $3,200 \mathrm{~mA} \mathrm{~g}^{-1}$, respectively. When the current density is turned back to $100 \mathrm{~mA} \mathrm{~g}^{-1}$, the capacity can recover $1,150 \mathrm{~mA} \mathrm{~h} \mathrm{~g}^{-1}$, showing a good tolerance for the rapid $\mathrm{Na}^{+}$insertion and extraction. Fig. $5 \mathrm{c}$ shows the selected charge-discharge curves at different current rates. Two discharge platforms could be observed throughout the discharge process. The initial voltage plateaus in the profile agree well with the peak positions in the $\mathrm{CV}$ curves. There is a moderate increase in polarization even at high current rate of $3,200 \mathrm{~mA} \mathrm{~g}^{-1}$, suggesting good electrical connectivity between the sulphur and carbon skeleton. As for the prominent capacity, it can be inferred from the above results that covalent-S cleaves from the carbon skeleton at lower potential stage and leaves lots of defects. Cleaved covalent- $S$ is precipitated in the isomerized carbon matrix that enables the reversible $S \rightarrow$ $\mathrm{S}_{x}^{2-} \rightarrow \mathrm{S}^{2-}$ reaction to occur at 0.6-2.8 V. Meanwhile, part of these defects are too small to allow $\mathrm{Na}^{+}$ions to pass through, prohibiting the larger species $\left(\mathrm{S}_{x}^{2-}\right)$, which is beneficial to reducing the polysufide shuttle effect $[35,36]$.
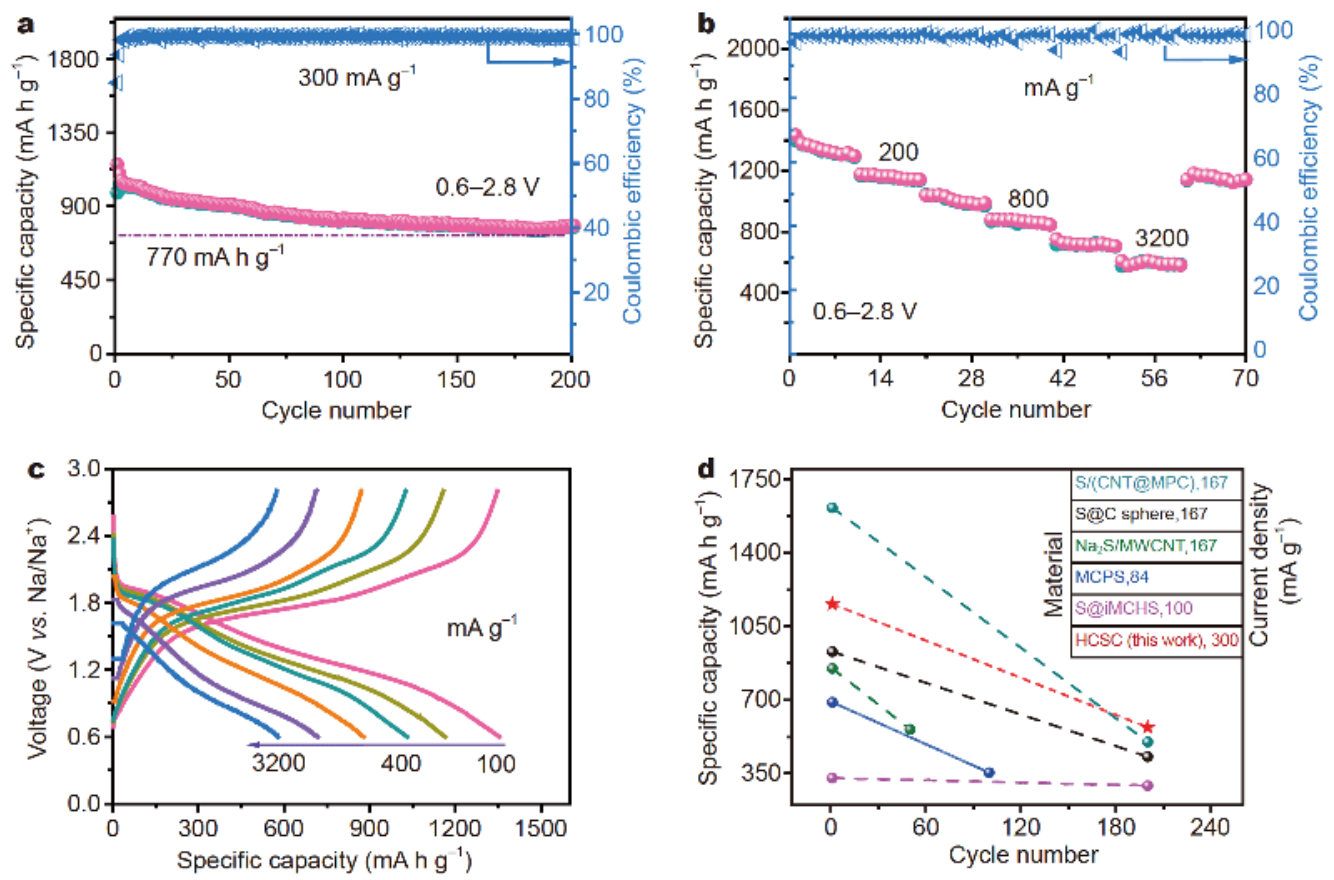

Figure 5 Electrochemical behaviors of HCSC electrode in the Na-S battery. (a) Cycling performance of the electrochemically activated HCSC in the $\mathrm{Na}-\mathrm{S}$ battery system (based on the mass of sulfur). (b) Rate performance of activated HCSC in the range of 100 to $3,200 \mathrm{~mA} \mathrm{~h} \mathrm{~g}^{-1}$. (c) Two discharge platforms could be observed throughout the discharge process in the corresponding charge-discharge profiles. (d) Comparison of electrochemical performances of different sulfur-doped carbon materials (first cycle $\rightarrow$ last cycle): S/(CNT@MPC) [11], S@C sphere [33], Na 2 S/MWCNT [34], MCPS [35], S@iMCHS [8], and HCSC (this work). The results show that the activated HCSC electrode displays higher reversible capacity and better cycling properties. 


\section{Investigation of bond evolution mechanism by in-situ XRD and Raman}

Firstly, to probe the structural evolution of HCSC sodiation/desodiation, we obtained the synchrotron-based XRD patterns of the electrode in operando. Beryllium foil served as the transition window to allow X-ray passage, and carbon paper acted as a current collector. The evolution of XRD patterns indicates a typical graphite behavior (carbon paper) during the initial period of sodium insertion and extraction (Fig. 6a) [37,38]. During the initial period of sodiation, graphite undergoes a onephase-like transformation. Gradually, the main peak at $27^{\circ}$ splits into two peaks (located at $23.6^{\circ}$ and $30.2^{\circ}$, re- spectively) and a biphasic reaction begins to occur. In the process of desodiation, the multiple new phases also involve one- or two phase reactions. Notably, after a cycle, the difference could be observed between the pristine state and the final desodiation state. Two new weak peaks gradually appear at $\sim 17.5^{\circ}$ and $\sim 19.2^{\circ}$ at the end of charging stage, which can be assigned to $\mathrm{Na}_{2} \mathrm{~S}_{x}$ and element sulfur $[39,40]$.

Next, in-situ Raman was also performed on a live cell with the HCSC anode operated over a voltage window of 0.01-3.0 V. The in-situ Raman provides direct evidences for the as-formed new species on the electrode surface, and the results are shown in Fig. 6b. At the initial stage of
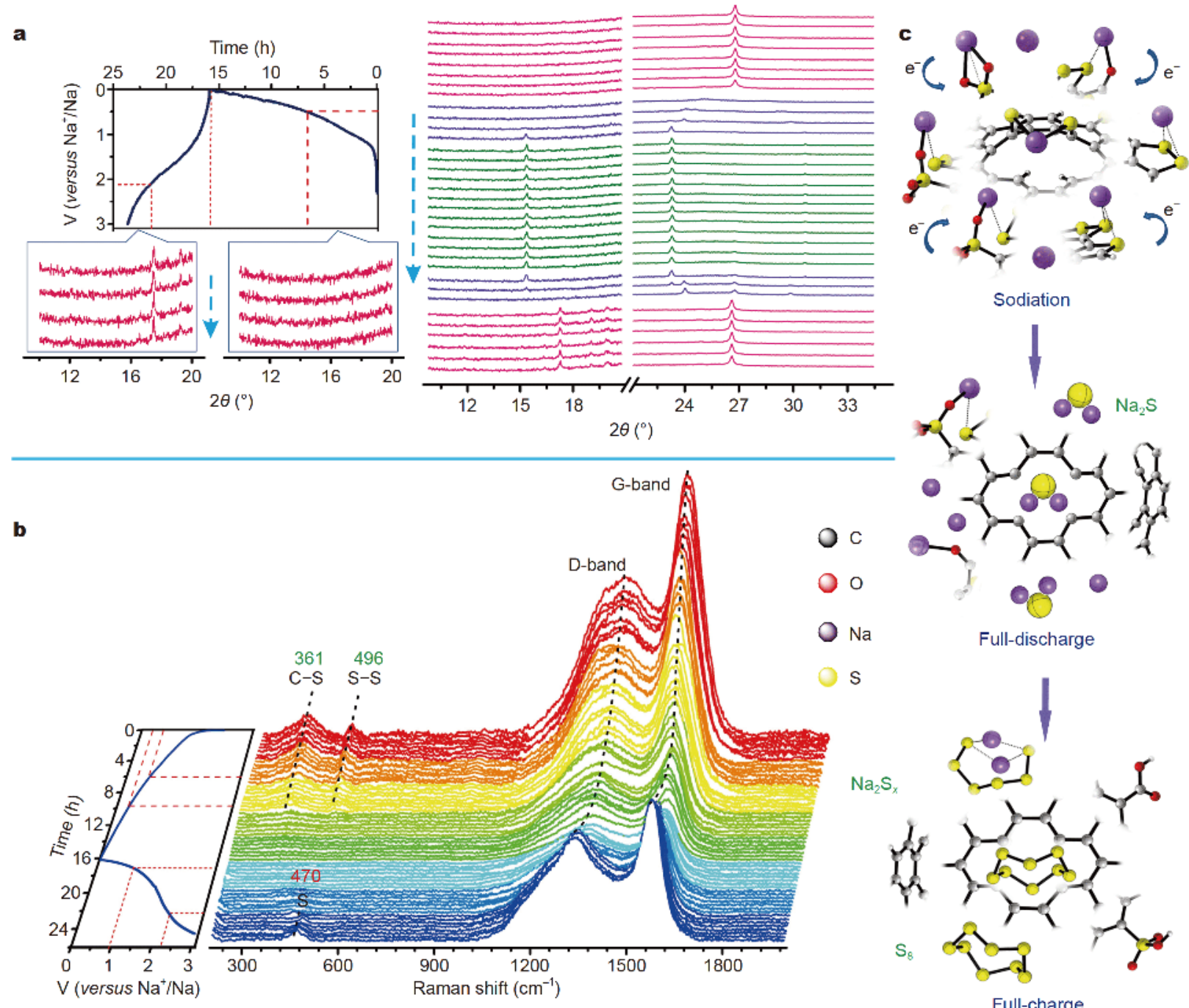

$\mathrm{s}$

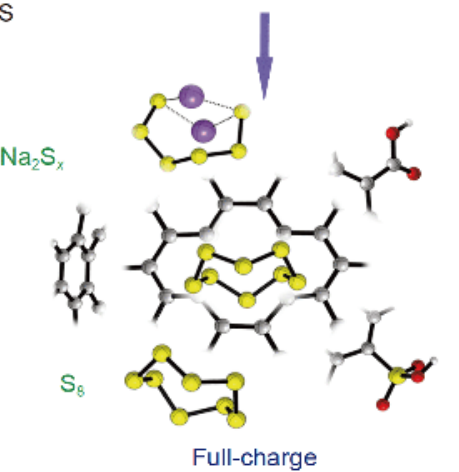

Figure 6 Investigation of the bond evolution mechanism by in-situ XRD and Raman. (a) In-situ XRD measurements of the reaction process. (b) Waterfall representation of Raman patterns and the corresponding galvanostatic curve. In-situ Raman records for the HCSC electrode through a discharge-charge cycle, showing the disappearance of C-S and S-S bonds and the formation of elemental sulfur. (c) Detailed schematic representation of the first discharge/charge cycle. 
sodiation (open circuit voltage to $0.7 \mathrm{~V}$ ), no obvious change is observed for the peaks of C-S-C and C-S-S-C. As the discharge voltage is continually scanned to lower than $\sim 0.4 \mathrm{~V}$, the two peaks gradually disappear, suggesting that most of the $\mathrm{C}-\mathrm{S}-\mathrm{C}$ and $\mathrm{C}-\mathrm{S}-\mathrm{S}-\mathrm{C}$ bonds are experiencing a substantial bond scission with the formation of sulfides during the discharge process. It is also apparent that the intensity of D and G-bands are slowly decreasing upon intercalation of $\mathrm{Na}^{+}$, which is well consistent with the previously reported literature [1]. When the HCSC anode is recharged to $2.6 \mathrm{~V}$, a new smooth peak is observed at $470 \mathrm{~cm}^{-1}$ and a gentle slope appears at about $200 \mathrm{~cm}^{-1}$, demonstrating that the linear polysulfides $\left(\mathrm{Na}_{2} \mathrm{~S}_{x}\right)$ and elemental sulfur are formed at the end of oxidation process. Meanwhile, the peaks of $\mathrm{D}$ and G bands have shown a prominent increase, where the $I_{\mathrm{D}} /$ $I_{\mathrm{G}}$ ratio is lower than the initial value, suggesting that the carbon skeleton has been irreversibly isomerized, and they cannot return to the original state. These results indicate that most of the chemical refined-S is ultimately cleaved from the carbon matrix with the intercalation of sodium ions at lower potentials, and $\mathrm{Na}_{2} \mathrm{~S}$ is simultaneously generated. In the following oxidation process, the lower order sulfides are gradually transferred into higher order linear polysulfides and elemental sulfur, and thus the carbon skeleton has undergone an irreversible isomerization. The corresponding schematic diagram of the electrochemical reaction process for the first discharge and charge is depicted in Fig. 6c. The HCSC mainly contains $\mathrm{C}-\mathrm{S}-\mathrm{C}$ and $\mathrm{C}-\mathrm{S}-\mathrm{S}-\mathrm{C}$ building blocks as well as some other functional groups $\left(-\mathrm{COOH},-\mathrm{SO}_{3} \mathrm{H}\right)$. In the sodiation process, these parts get electrons $\left(\mathrm{e}^{-}\right)$, and then gradually combine with $\mathrm{Na}^{+}$, which finally form the $\mathrm{Na}_{2} \mathrm{~S}$ and isomerized carbon in the full-discharge stage. In the following charge process, $\mathrm{Na}_{2} \mathrm{~S}$ is gradually transferred into sodium polysulfide $\left(\mathrm{Na}_{2} \mathrm{~S}_{x}\right)$ and elemental $\mathrm{S}$, showing the behavior of $\mathrm{Na}-\mathrm{S}$ batteries.

\section{Investigation of reaction mechanism by $e x$-situ techniques} These aforementioned results prompt us to explore the intermediates during the first charge/discharge process, which is very helpful to ultimately verify the electrochemical reaction mechanism of HCSC electrode. Firstly, XPS was employed to characterize the HCSC intermidiate electrode after being discharged to $0.6 \mathrm{~V}$ (Fig. 7a). Compared with the initial HCSC electrode, two dominate peaks (163.7 and $164.8 \mathrm{eV}$ ) for C-S and S-S bridged bonds still stably exist in the $\mathrm{S} 2 \mathrm{p}$ spectra. However, $\mathrm{Na}^{+}$ intercalates into HCSC (Fig. S9a) and a small broad peak appears at $162.0 \mathrm{eV}$ that could be assigned to sulfides
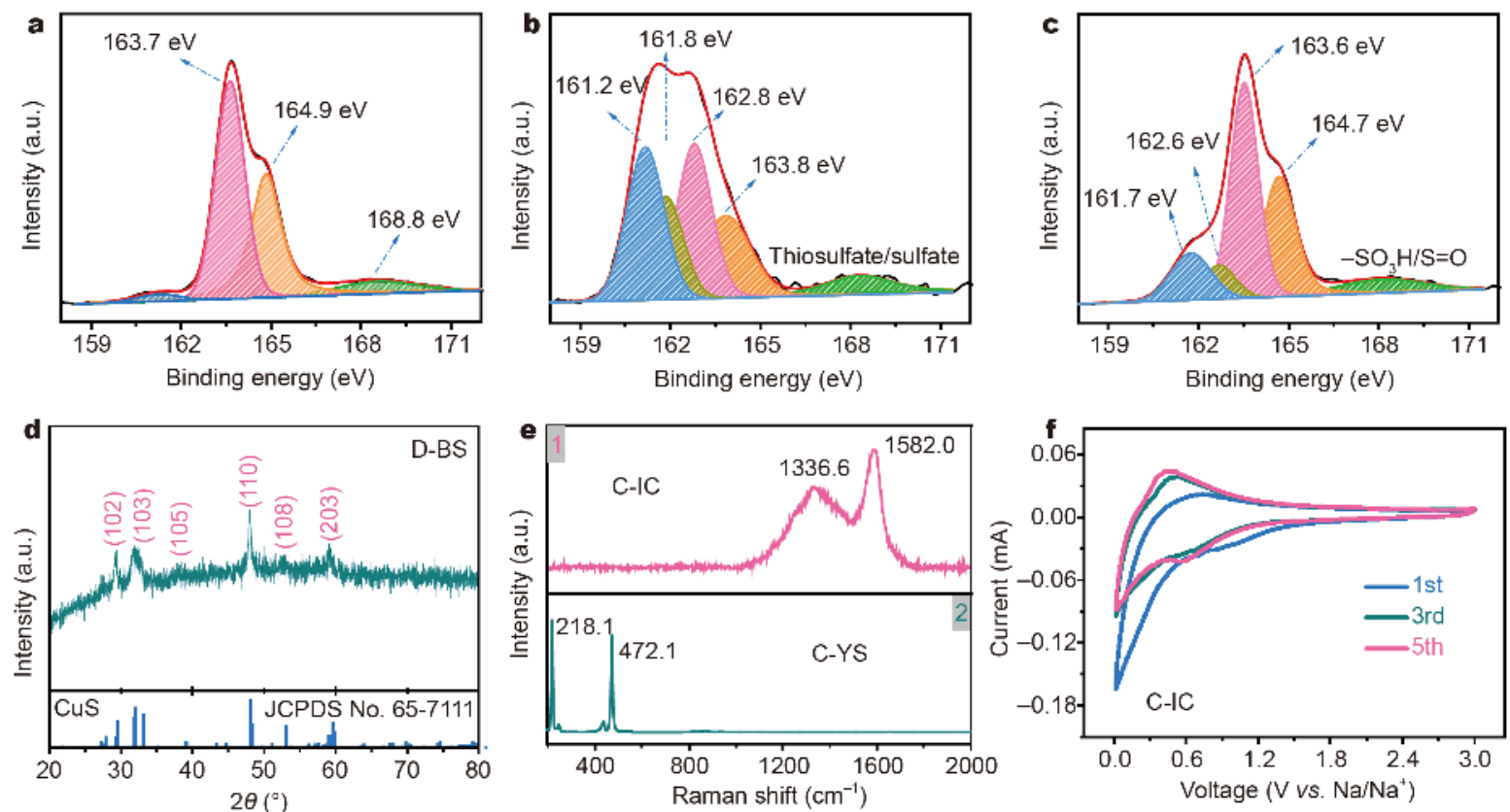

Figure 7 Investigation of the reaction mechanism by ex-situ techniques. (a-c) XPS analysis of the S 2p peaks from HCSC electrode at different discharge/charge states: (a) first discharge to $0.6 \mathrm{~V}$; (b) first discharge to $0.01 \mathrm{~V}$; (c) first charge to $3.0 \mathrm{~V}$. (d) XRD pattern of D-BS that confirms the $\mathrm{S}^{2-}$ in the fully-discharged electrode. (e) Raman spectra of C-IC (1) and C-YS (2) indicate that the isomerized carbon and elemental sulfur are produced at the final charged stage. (f) CV profiles of C-IC. 
[12]. It means that the chemical refined-S within the HCSC surface has not obviously changed in the initial reduction process.

Next, to further confirm the final products at the end of reduction process $(0.01 \mathrm{~V})$, the as-obtained discharged electrode was similarly analyzed by XPS. As shown in Fig. $7 \mathrm{~b}$, the XPS peaks of chemical refined-S have consequently disappeared and new peaks at 161.2/161.8 and $162.8 / 163.8 \mathrm{eV}$ are displayed in the S $2 \mathrm{p}$ spectra, showing that the chemical refined-S has been electrochemically reduced to sulfides. At last, when the charge potential reaches $3.0 \mathrm{~V}$ (Fig. $7 \mathrm{c}$ ), the $S 2 p_{3 / 2}$ and $S 2 p_{1 / 2}$ peaks are shifted positively to 162.0 and $163.5 \mathrm{eV}$, respectively, with an additional peak being presented at 161.7/162.6 and $163.6 / 164.7 \mathrm{eV}$, which can be attributed to the polysulfides and elemental sulfur [11].

To further complement the XPS results, the ex-situ techniques were employed to capture the intermediates. At first, the fully discharged electrode slices were extracted with distilled water, and the extract liquor was mixed with $\mathrm{CuSO}_{4}$ solution to form black solid (D-BS). Not surprisingly, the XRD result of D-BS matches well with CuS (JCPDS No. 65-7111), demonstrating the existence of $\mathrm{S}^{2-}$ at the fully-discharged stage (Fig. $7 \mathrm{~d}$ and Fig. S9e). Secondly, the fully-charged electrode was extracted in chloroform, and then the resultant solution was dried and purified, resulting in a yellow solid (C-YS). The Raman spectrum of C-YS indicates that the elemental sulfur is present (Fig. 7e-2). Furthermore, the extracted residue is the isomerized carbon (C-IC), and the surface atomic chemical refined-S is only about 1.18 wt.\% (Fig. S9c, d). And the CV curves of C-IC electrode show no obvious oxidation/reduction platforms at $0.01-3.0 \mathrm{~V}$ (Fig. 7 f and Fig. S9b). The $I_{\mathrm{D}} / I_{\mathrm{G}}$ ratio $(0.68)$ is higher than that of the fresh HCSC electrode in the Raman spectrum (Fig. 7e-1). The above analyses further supplement the XPS results: $\mathrm{Na}_{2} \mathrm{~S}$ is produced at the fully-discharged state, while the elemental sulfur is yielded at nearly fullycharged state, accompanying with the irreversible isomerism of the carbon skeleton.

\section{CONCLUSIONS}

In summary, we reported a new strategy to prepare the sulfurized-carbon bridged complex HCSC with phenylphosphinic acid and sodium sulfate as the starting materials. No elemental sulfur was used in the synthesis, and the HCSC contained 34.8 wt.\% chemical refined-S. Its structural benefits as anodes were evaluated in a half-cell system, which exhibited unique electrochemical properties at various voltage ranges. The material structure changes during the first discharging and charging process were directly monitored by in-situ XRD and Raman, in which the irreversibly evolution of C-S, S-S, and carbon skeleton were observed, and meanwhile, some characteristic peaks of sulfide were also detected. In addition, ex-situ techniques were also employed to explore the intermediates in the first cycle. At last, the $\mathrm{S}^{2-}$ and C-IC were captured in the final discharged state, and the elemental sulfur was extracted in the full charged status. The electrochemical reaction mechanism of HCSC electrode was ultimately verified-when the negative scan was gradually further swept down to $0.01 \mathrm{~V}$, the bridged sulfur was gradually broken away from the carbon skeleton with simultaneous generation of $\mathrm{Na}_{2} \mathrm{~S}$, which was then progressively oxidized to elemental $\mathrm{S}$ in the following charge process. Thus, the HCSC electrode was activated in $0.01-3.0 \mathrm{~V}$, and then applied in the Na-S battery system $(0.6-2.8 \mathrm{~V})$. It showed superior electricalchemical performance $\left(770 \mathrm{~mA} \mathrm{~h} \mathrm{~g}^{-1}\right.$ over 200 cycles at $300 \mathrm{~mA} \mathrm{~g}^{-1}$ and $590 \mathrm{~mA} \mathrm{~h} \mathrm{~g}^{-1}$ at $3,200 \mathrm{~mA} \mathrm{~g}^{-1}$ ). The analogous electrochemical phenomenon was also noticed at different potential window versus $\mathrm{Li} / \mathrm{Li}^{+}$. This strategy can be applied in other chemical refined materials to provide a route for the essential effects of chemical bonds on electrochemical properties in rechargeable batteries.

Received 20 February 2019; accepted 15 March 2019; published online 10 April 2019

1 Guo Q, Ma Y, Chen T, et al. Cobalt sulfide quantum dot embedded N/S-doped carbon nanosheets with superior reversibility and rate capability for sodium-ion batteries. ACS Nano, 2017, 11: 1265812667

2 Li Z, Chen Y, Jian Z, et al. Defective hard carbon anode for Na-ion batteries. Chem Mater, 2018, 30: 4536-4542

3 Xin $\mathrm{S}, \mathrm{Gu} \mathrm{L}$, Zhao $\mathrm{NH}$, et al. Smaller sulfur molecules promise better lithium-sulfur batteries. J Am Chem Soc, 2012, 134: 1851018513

4 Tan G, Xu R, Xing Z, et al. Burning lithium in $\mathrm{CS}_{2}$ for highperforming compact $\mathrm{Li}_{2} \mathrm{~S}$-graphene nanocapsules for $\mathrm{Li}-\mathrm{S}$ batteries. Nat Energy, 2017, 2: 17090

5 Fan L, Ma R, Yang Y, et al. Covalent sulfur for advanced room temperature sodium-sulfur batteries. Nano Energy, 2016, 28: 304-310

6 Yang CP, Yin YX, Guo YG, et al. Electrochemical (de)lithiation of 1D sulfur chains in Li-S batteries: a model system study. J Am Chem Soc, 2015, 137: 2215-2218

7 Yang J, Zhou X, Wu D, et al. S-doped N-rich carbon nanosheets with expanded interlayer distance as anode materials for sodiumion batteries. Adv Mater, 2017, 29: 1604108

$8 \mathrm{Xu} \mathrm{D}$, Chen C, Xie J, et al. A hierarchical N/S-codoped carbon anode fabricated facilely from cellulose/polyaniline microspheres for high-performance sodium-ion batteries. Adv Energy Mater, 2016, 6: 1501929

9 Wang YX, Yang J, Lai W, et al. Achieving high-performance roomtemperature sodium-sulfur batteries with S@interconnected me- 
soporous carbon hollow nanospheres. J Am Chem Soc, 2016, 138: 16576-16579

10 Qie L, Chen W, Xiong X, et al. Sulfur-doped carbon with enlarged interlayer distance as a high-performance anode material for sodium-ion batteries. Adv Sci, 2015, 2

11 Tuček J, Błoński P, Sofer Z, et al. Sulfur doping induces strong ferromagnetic ordering in graphene: effect of concentration and substitution mechanism. Adv Mater, 2016, 28: 5045-5053

12 Xin S, Yin YX, Guo YG, et al. A high-energy room-temperature sodium-sulfur battery. Adv Mater, 2014, 26: 1261-1265

13 Wang YX, Zhang B, Lai W, et al. Room-temperature sodiumsulfur batteries: a comprehensive review on research progress and cell chemistry. Adv Energy Mater, 2017, 7: 1602829

14 Conder J, Bouchet R, Trabesinger S, et al. Direct observation of lithium polysulfides in lithium-sulfur batteries using operando Xray diffraction. Nat Energy, 2017, 2: 17069

$15 \mathrm{Yu}$ X, Manthiram A. Performance enhancement and mechanistic studies of room-temperature sodium-sulfur batteries with a carbon-coated functional nafion separator and a $\mathrm{Na}_{2} \mathrm{~S} /$ activated carbon nanofiber cathode. Chem Mater, 2016, 28: 896-905

16 Wang X, Li G, Hassan FM, et al. Sulfur covalently bonded graphene with large capacity and high rate for high-performance sodium-ion batteries anodes. Nano Energy, 2015, 15: 746-754

17 Poh HL, Šimek P, Sofer Z, et al. Sulfur-doped graphene via thermal exfoliation of graphite oxide in $\mathrm{H}_{2} \mathrm{~S}, \mathrm{SO}_{2}$, or $\mathrm{CS}_{2}$ gas. ACS Nano, 2013, 7: 5262-5272

18 Graczyk PP, Mikolajczyk M. Inapplicability of the antiperiplanar lone pair hypothesis to $\mathrm{C}-\mathrm{P}$ bond breaking and formation in some S-C- $\mathrm{P}^{+}$systems. J Org Chem, 1996, 61: 2995-3002

19 Okuma K, Shigetomi T, Nibu Y, et al. Synthesis of isolable thiirane-2-thione ( $\alpha$-dithiolactone) from thioketene S-oxide. J Am Chem Soc, 2004, 126: 9508-9509

20 Juaristi E, Notario R. Theoretical examination of the S-C-P anomeric effect. J Org Chem, 2015, 80: 2879-2883

21 Legnani L, Toma L, Caramella P, et al. Computational mechanistic study of thionation of carbonyl compounds with Lawesson's reagent. J Org Chem, 2016, 81: 7733-7740

22 Yu X, Xie J, Yang J, et al. Lithium storage in conductive sulfurcontaining polymers. J Electroanal Chem, 2004, 573: 121-128

23 Sainbileg B, Lan YB, Wang JK, et al. Deciphering anomalous Raman features of regioregular poly(3-hexylthiophene) in ordered aggregation form. J Phys Chem C, 2018, 122: 4224-4231

24 Raccichini R, Varzi A, Passerini S, et al. The role of graphene for electrochemical energy storage. Nat Mater, 2014, 14: 271-279

25 Pachfule P, Shinde D, Majumder M, et al. Fabrication of carbon nanorods and graphene nanoribbons from a metal-organic framework. Nat Chem, 2016, 8: 718-724

26 Kumar R, Pillai RG, Pekas N, et al. Spatially resolved raman spectroelectrochemistry of solid-state polythiophene/viologen memory devices. J Am Chem Soc, 2012, 134: 14869-14876

27 Xu J, Wang M, Wickramaratne NP, et al. High-performance sodium ion batteries based on a 3D anode from nitrogen-doped graphene foams. Adv Mater, 2015, 27: 2042-2048

28 Ghanty TK, Ghosh SK. Simple density functional approach to polarizability, hardness, and covalent radius of atomic systems. J Phys Chem, 1994, 98: 9197-9201

29 Zhou G, Paek E, Hwang GS, et al. Long-life Li/polysulphide batteries with high sulphur loading enabled by lightweight three-dimensional nitrogen/sulphur-codoped graphene sponge. Nat Commun, 2015, 6: 7760
30 Shi X, Chen Y, Lai Y, et al. Metal organic frameworks templated sulfur-doped mesoporous carbons as anode materials for advanced sodium ion batteries. Carbon, 2017, 123: 250-258

31 Cao Y, Xiao L, Sushko ML, et al. Sodium ion insertion in hollow carbon nanowires for battery applications. Nano Lett, 2012, 12: 3783-3787

32 Ding J, Wang H, Li Z, et al. Carbon nanosheet frameworks derived from peat moss as high performance sodium ion battery anodes. ACS Nano, 2013, 7: 11004-11015

33 Carter R, Oakes L, Douglas A, et al. A sugar-derived room-temperature sodium sulfur battery with long term cycling stability. Nano Lett, 2017, 17: 1863-1869

$34 \mathrm{Yu}$ X, Manthiram A. $\mathrm{Na}_{2} \mathrm{~S}$-carbon nanotube fabric electrodes for room-temperature sodium-sulfur batteries. Chem Eur J, 2015, 21: 4233-4237

35 Wei S, Xu S, Agrawral A, et al. A stable room-temperature sodium-sulfur battery. Nat Commun, 2016, 7: 11722

36 Qiang Z, Chen YM, Xia Y, et al. Ultra-long cycle life, low-cost room temperature sodium-sulfur batteries enabled by highly doped (N,S) nanoporous carbons. Nano Energy, 2017, 32: 59-66

37 Kim H, Hong J, Yoon G, et al. Sodium intercalation chemistry in graphite. Energy Environ Sci, 2015, 8: 2963-2969

38 Placke T, Schmuelling G, Kloepsch R, et al. In situ X-ray diffraction studies of cation and anion intercalation into graphitic carbons for electrochemical energy storage applications. $\mathrm{Z}$ anorg allg Chem, 2014, 640: 1996-2006

39 Yu X, Manthiram A. Room-temperature sodium-sulfur batteries with liquid-phase sodium polysulfide catholytes and binder-free multiwall carbon nanotube fabric electrodes. J Phys Chem C, 2014, 118: 22952-22959

40 Park J, Yu BC, Park JS, et al. Tungsten disulfide catalysts supported on a carbon cloth interlayer for high performance Li-S battery. Adv Energy Mater, 2017, 7: 1602567

Acknowledgements This work was supported by the National Key Research and Development Program of China (2017YFB0102003 and 2018YFB0104204), the National Natural Science Foundation of China (51622406, 21673298 and 21473258), Young Elite Scientists Sponsorship Program By CAST (2017QNRC001), the Project of Innovation Driven Plan in Central South University (2017CX004 and 2018CX005), and the Program for Innovative Team (in Science and Technology) in the University of Henan Province of China (17IRTSTHN003). This work was carried out in part using hardware and/or software provided by Tianhe II super computer in the National Supercomputing Center in Guangzhou, and the High-Performance Computing Centers of Central South University and Nanjing University.

Author contributions $\mathrm{Wu} \mathrm{T}$ and Ji X conceived the idea, experiments, materials characterizations and electrochemical measurements. $\mathrm{Hu} \mathrm{J}$ conducted the in-situ Raman and XRD characterizations. Zhang C performed the first-principles calculations. Hou H and Zou G assisted the date analysis. Cao X co-wrote the paper. All authors discussed the results and commented on the manuscript.

Conflict of interest The authors declare that they have no conflict of interest.

Supplementary information Experimental details and supporting data are available in the online version of the paper. 


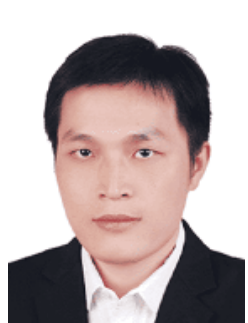

Tianjing Wu received his Master degree from Henan University in 2016. He is currently working toward a PhD degree in Central South University and his current research focuses on the energy storage materials and devices.

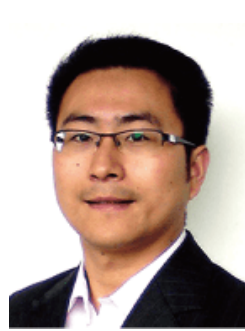

Xiaobo Ji is a "Shenghua" Professor at Central South University and a Fellow of the Royal Society of Chemistry, specializing in the research and development of batteries and supercapacitor materials and their systems. He received his $\mathrm{PhD}$ degree in electrochemistry in 2007 under the supervision of Prof. Richard Compton at the University of Oxford and undertook postdoctoral work at MIT with Prof. Donald Sadoway.

\section{共价硫碳材料在储能过程中的价键演变机制}

吴天景 ${ }^{1}$, 张晨阳 ${ }^{1}$, 邹国强, 胡久刚 ${ }^{1}$, 朱利敏 ${ }^{2}$, 曹晓雨 ${ }^{2 *}$, 侯红帅 ${ }^{1}$, 纪效波 ${ }^{1 *}$

摘要 共价硫碳材料优异的储能性能逐渐引起人们的极大关注, 然 而, 在电化学钠储存过程中, 化学键的演变机制尚不清楚. 本文以苯 基磷酸作为碳源和催化剂, 硫酸钠为硫源和模板, 通过高温热处理, 成功制备了具有大量共价键的硫碳材料(HCSC), 其中硫主要以CS-C和C-S-S-C的短链形式存在. 值得注意的是, 在储钠过程中, 当 循环电压低于 $0.6 \mathrm{~V}$ 时, 大多数桥键会发生电化学裂解, 导致在接下 来的 CV 测试中出现了两个可见的氧化还原峰. 原位和非原位测试 表明, 在还原过程中形成了 $\mathrm{S}^{2-}$, 同时碳骨架也发生了不可逆的异构 化. 因此, 在接下来的循环过程中 $(0.01-3.0 \mathrm{~V})$, 裂解硫和异构化碳 可以共同参与钠的存储. 同样, 应用于 $\mathrm{Na}-\mathrm{S}$ 电池系统中, 电压窗口 为0.6-2.8 V, 在宽电压窗口活化的HCSC也表现出较高的可逆容量 $\left(770 \mathrm{~mA} \mathrm{~h} \mathrm{~g}^{-1}\right.$ at $\left.300 \mathrm{~mA} \mathrm{~g}^{-1}\right)$. 这一发现揭示了硫碳桥联化合物的 储能机理, 也为其他电极材料的表界面化学提供了新的启示. 